\title{
Systematically Creased Thin-Film Membrane Structures
}

\author{
Alessandro Papa* and Sergio Pellegrino亡 \\ University of Cambridge, \\ Cambridge, England CB2 1PZ, United Kingdom
}

DOI: $\underline{10.2514 / 1.18285}$

\begin{abstract}
This paper presents a study of a square membrane, creased according to the Miura-ori folding pattern. When the membrane is allowed to expand from its packaged configuration, it initially expands elastically under zero corner forces. Starting from this naturally expanded configuration, the paper investigates the stress distribution and the load-displacement relationship when in-plane, diagonal loads are applied at the corners. It is found that out-of-plane bending is the main load-carrying mode and, for stress magnitudes typical of current solar-sail designs, the behavior of the membrane remains linear elastic. A simple analytical model, originally proposed for randomly creased membranes, is shown to predict with good accuracy the load-displacement relationship of the corners. It uses physically based and hence directly measurable membrane parameters.
\end{abstract}

\section{Introduction}

$\mathbf{T}$ HERE is currently much interest in the use of thin-film membrane structures for a variety of future gossamer spacecraft missions. In some of the proposed structural architectures, it is envisaged that thin films will be packaged by means of a regular arrangement of creases, or fold lines, and hence the question arises of how such precreased foils behave when they are deployed in orbit.

One packaging scheme is known as Miura-ori [1], Fig. 1. An ideal Miura-ori surface is a mechanical linkage of thin, flat plates connected by frictionless hinges; this linkage has a single degree of kinematic freedom. A membrane folded such that the pattern of the resulting creases mimics the arrangement of the hinges in an ideal Miura-ori surface can be packaged and deployed efficiently [2,3]. Because of these properties, the Miura-ori crease pattern was selected for the present research.

This paper presents a detailed study (involving both experiments and detailed finite element simulations) of a square membrane with specific dimensions and creases arranged according to the Miura-ori folding pattern. Randomly creased thin films have been extensively investigated by Murphey [4], and the present study is an extension of Murphey's work to films where the creases are arranged systematically, according to a repeating pattern. The aim of our study is to determine the shape of the membrane and the loaddisplacement relationship for in-plane, diagonal loading of the corners, starting from a configuration that might be considered as the unstressed deployed shape of the membrane, i.e., the configuration that the membrane will expand to, in the absence of any external forces. The magnitudes of the corner forces are chosen such as to generate stress levels on the order of $0.02 \mathrm{MPa}$ at the center of a $25 \mu \mathrm{m}$ thick membrane. We show that a simple analytical model consisting of two creased beams, derived from Murphey's model for randomly creased membranes and characterized by only a small

Presented as Paper 1975 at the 46th AIAA/ASME/ASCE/AHS/ASC Structures, Structural Dynamics and Materials Conference, Austin, Texas, 18-21 April 2005; received 18 June 2005; revision received 11 September 2006; accepted for publication 16 September 2006. Copyright () 2007 by Alessandro Papa and Sergio Pellegrino. Published by the American Institute of Aeronautics and Astronautics, Inc., with permission. Copies of this paper may be made for personal or internal use, on condition that the copier pay the $\$ 10.00$ per-copy fee to the Copyright Clearance Center, Inc., 222 Rosewood Drive, Danvers, MA 01923; include the code 0022-4650/08 $\$ 10.00$ in correspondence with the CCC.

${ }^{*}$ Mechanical Engineering Student, Department of Engineering, Trumpington Street; Alessandro.Papa@ cantab.net.

${ }^{\dagger}$ Professor of Structural Engineering, Department of Engineering, Trumpington Street; currently Professor of Aeronautics and Civil Engineering, California Institute of Technology, 1200 East California Boulevard, Mail Code 301-46, Pasadena, CA 91125; sergiop@ caltech.edu. Associate Fellow AIAA. number of directly measurable parameters, predicts the displacements of the corners quite accurately.

The paper is laid out as follows. Section II describes the layout of the creases. Section III presents an experimental study of a $0.5 \times$ $0.5 \mathrm{~m}$ membrane creased according to the Miura-ori pattern. The experimental technique is explained and measurements of the initial crease angle are obtained. Section IV presents a detailed finite element simulation of this membrane. Section $\underline{V}$ compares experimental measurements with finite element results. Finally, Sec. VI presents a simple analytical model for the membrane's loaddisplacement behavior, whose predictions are then compared with the finite element results. Section VII concludes the paper.

\section{Crease Geometry}

There are two sets of creases, as shown in Fig. 1. Those in the first set, called primary creases, form the main hill and valley folds of the packaging scheme; each of these creases is folded in the same sense throughout its length, and it never becomes straight. The creases in the second set, called secondary creases, form alternate hill and valley folds and become aligned when the membrane is flat. A detail of the crease geometry on a flat membrane is shown in Fig. 2a.

The degree of freedom used to describe the degree of deployment of a Miura-ori surface is the deployment angle $\theta$, defined as the angle between the panels and the plane $x-y$ of the fully deployed surface (see Fig. 2b).

The geometric feature that determines the deployment path, and therefore the degree of coupling between the expansion of the membrane along the two diagonal directions, is the defect angle $\beta$, defined in Fig. 2a. The expansion ratios of a Miura-ori surface, in the $x$ and $y$ directions, are related to $\theta$ by [1]

$$
\begin{aligned}
\frac{\overline{O A}}{\overline{O A_{\max }}}=\cos \left[\sin ^{-1}(\sin \theta \cos \beta)\right] \\
\frac{\overline{O B}}{\overline{O B}_{\max }}=\frac{\cos \theta}{\cos \left[\sin ^{-1}(\sin \theta \cos \beta)\right]}
\end{aligned}
$$

\section{Experimental Study}

The experimental study had two principal aims. First, to obtain a representative value for the initial deployment angle $\theta_{0}$ to be used in the finite element simulations. Second, to measure the loaddisplacement relationship for the corners of the membrane and so test the validity of the elastic behavior assumption. 


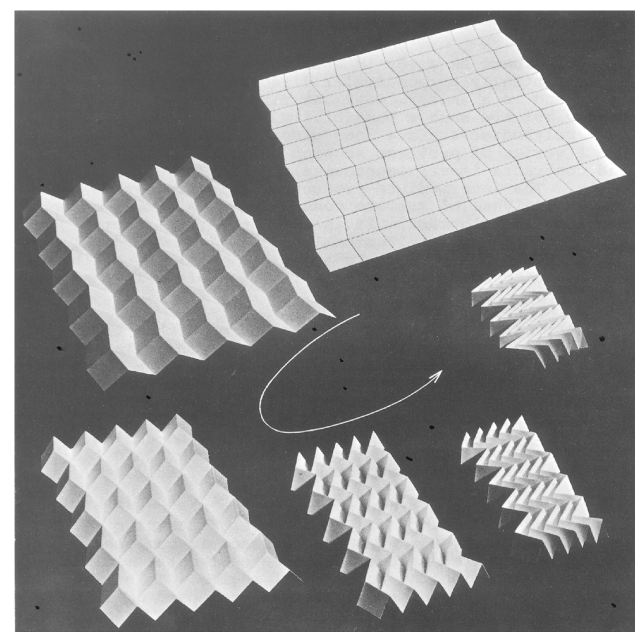

Fig. 1 Miura-ori packaging scheme [1]
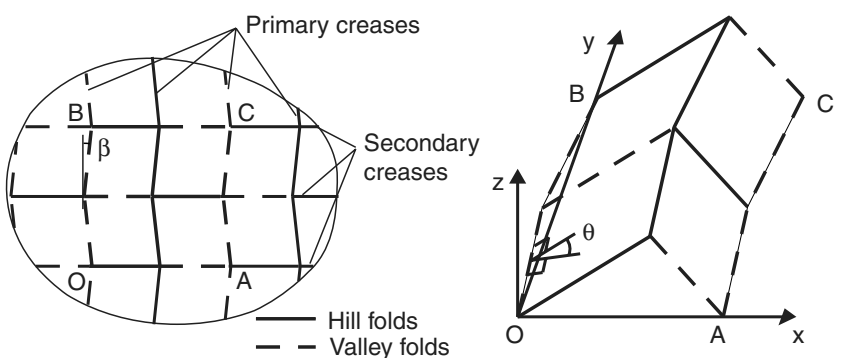

a) Flat membrane

b) Perspective view

Fig. 2 Details of crease geometry, defect angle $\beta$, and deployment angle $\theta$.

\section{A. Membrane}

A $25 \mu \mathrm{m}$ thick aluminized Kapton membrane was used. The required crease pattern was set up as follows. A $5 \times 5$ panel Miura-ori crease pattern was marked with a fine permanent marker on one side of a $500 \times 500 \mathrm{~mm}$ square membrane. The secondary convex creases, along the $x$ direction, were formed by running a ballpoint pen firmly along the marked crease lines. The membrane was then turned and the secondary concave creases formed in a similar fashion. By placing the membrane over a compliant surface consisting of a dozen sheets of A4 paper and carefully controlling the applied force, a homogenous set of secondary creases was formed.

For an ideal Miura-ori surface at small angles of deployment, the amplitude of the creases in the $x$ direction is considerably larger than the amplitude of the creases in the $y$ direction. To allow for this difference, the primary creases were formed using a different technique to that used to form the secondary creases. A thin plate of Kevlar was cut into a rectangular shape, with the length of its minor edge matching the length of the primary creases. For each primary crease, the minor edge of the Kevlar plate was placed to coincide with its desired position and the membrane carefully folded 180 deg over the Kevlar plate, avoiding any damage to the membrane. A roller was then run along the length of the Kevlar edge, through the folded membrane, thereby forming a tight crease in the membrane. This process was repeated until the crease pattern was complete.

\section{B. Apparatus}

A detailed description of the rig used for these experiments can be found in [5]. The creased membrane can be seen in Fig. 3, attached to a horizontal steel frame. Note that the corners have been cut at an angle and reinforced with edge tabs, made of Kapton tape looped over a steel rod. These corner reinforcements have the purpose of reducing the maximum stress in the membrane and so avoid plastic deformation in the corner regions.

The forces applied to the corners of the membrane, which ranged from 0 to $0.2 \mathrm{~N}$, were measured with strain gauged cantilever beams

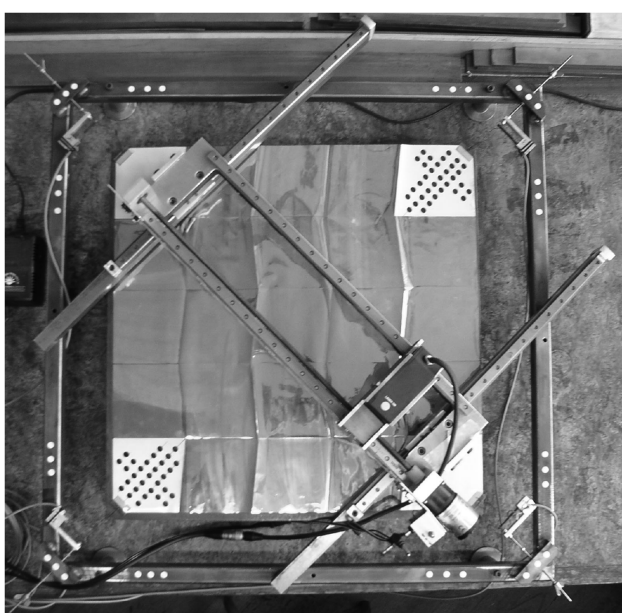

Fig. 3 Test rig, including CCD laser, cantilever strain gauges, and target markers.

tied with a light Kevlar cord to a small hole in the membrane corner tab. Given that the membrane's total weight is approximately $0.11 \mathrm{~N}$, the experiment had to be carried out with the membrane supported on a flat surface. A steel plate was placed on wood block supports in the interior of a square steel frame, such that its top surface was at the same height as the strain gauged cantilevers attached to the corners of the steel frame, as shown in Fig. 3.

A Keyence LK-081 charge-coupled device (CCD) laser was mounted to the steel rig so that it could scan the membrane and measure its surface profile. The resolution of this laser is $3 \mu$ m over a range of $\pm 15 \mathrm{~mm}$.

Photogrammetry was used to track the relative displacements of two opposite corners of the membrane. Thirty black markers were affixed to the two corners of the membrane (which had previously been covered with a thin coat of white spray) and nine white markers were affixed to each corresponding corner of the rig; all of these targets were paper disks with a diameter of $9 \mathrm{~mm}$. Photographs of the complete membrane were taken with a 4.0 megapixel digital camera, and the images were processed with the software Photomodeler Pro 4.0. The average standard deviation of the measurements was $0.11 \mathrm{~mm}$, or $6.0 \%$ of the maximum displacement measured.

\section{Surface Profile}

The average crease angle of the membrane in the unloaded state was measured to be $\eta_{0}=152.4 \mathrm{deg}$. Hence the corresponding value of $\theta_{0}$, see Fig. $\underline{4}$, is

$$
\theta_{0}=\frac{180-\eta_{0}}{2}=13.8 \mathrm{deg}
$$

The average crease angle of the membrane in the fully loaded state was measured as $\eta_{1}=156.0 \mathrm{deg}$; this is a $2.4 \%$ increase from $\eta_{0}$.

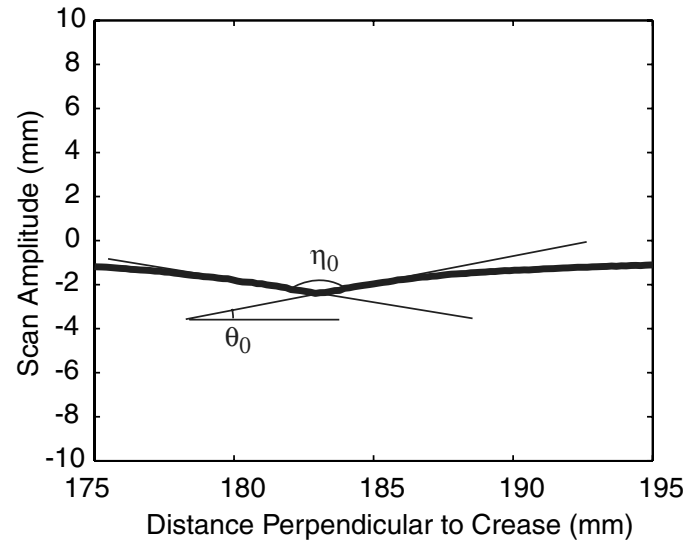

Fig. 4 Measured profile of a primary crease. 
The measured load-displacement relationship is presented in Sec. V.

\section{Finite Element Analysis}

Detailed finite element simulations, based on shell-element models of a membrane, have already been used extensively to study the phenomenon of two-dimensional structural wrinkling in initially flat thin-film membranes [6-8]. The present study represents the first attempt to apply the same approach to the study of precreased membranes. A parallel effort, which uses a nonlinear material model for a creased membrane, has been recently presented [9].

The finite element model aimed to reproduce the behavior of the $5 \times 5$ panel experimental membrane described in Sec. III. Hence all internal panels form identical parallelograms with $\beta=6 \mathrm{deg}$, as in the experiment. To set up the model, it was assumed that a thin-film membrane with uniform Miura-ori creases opens out elastically and takes the shape of an ideal Miura-ori surface, with flat panels joining the creases.

\section{A. Finite Element Mode}

The ABAQUS finite element package (version 6.4) was used for this study; the geometry of the model is shown in Fig. 5. The membrane and the corner tabs were modeled with S4R $\overline{5}$ shell elements. S4R5 is a four-node, doubly curved, reduced integration, thin-shell element, with five degrees of freedom per node. B31 beam elements were used to model the rods through which the corner loads are applied. B31 is a two-node linear beam element. The corner nodes were constrained as follows: node $\mathrm{A}$ in the $x, y$, and $z$ directions; node $\mathrm{B}$ in $x$ and $z$; node $\mathrm{C}$ in $z$ only; and node $\mathrm{D}$ in $y$ and $z$. These constraints were representative of the boundary conditions that would be applied to a real solar sail; convergence could not be achieved when fewer constraints were applied.

A MATLAB script was developed to determine the coordinates of a mesh of evenly distributed nodes lying on an ideal Miura-ori surface, at any stage of deployment. The edges of each panel of the membrane are defined exactly, by defining sets of nodes that lie on the crease lines. Given $\theta_{0}$, from Eq. (3), this script was used to generate the ABAQUS input file.

\section{B. Material Properties}

It was assumed that Kapton behaves as a linear-elastic material for the load range of interest. Note that the initial formation of creases in

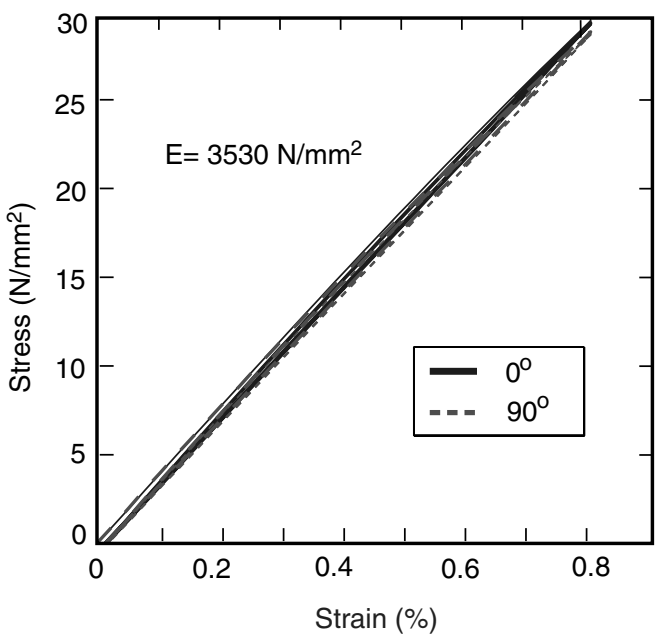

Fig. 6 Behaviour of $25 \mu \mathrm{m}$ Kapton $\mathrm{HN}$ in uniaxial tension; for each orientation, three load-unload cycles were carried out [10].

the membrane, which involves localized yielding of the material along a line, cannot be captured (of course) by this approach. Because the analysis assumes an initial set of crease lines in the membrane, with associated kinks, the assumption of linear-elastic behavior amounts to assuming that no plastic deformation takes place, even along the creases, under the action of the applied corner loads.

A detailed set of measurements of the stress-strain behavior of the same material used in the present research, Kapton HN, were carried out by Wong [10]. These tests showed that at room temperature this material is almost perfectly linear elastic, see Fig. $\underline{6}$, for stresses below $30 \mathrm{MPa}$. This value is about 10 times larger than the largest stress induced by the load magnitudes of interest; we will return to this point in Sec. IV.G.

Based on Wong's results, the Kapton membrane was modeled as an isotropic, linear-elastic material with Young's modulus $E=$ $3530 \mathrm{MPa}$ and Poisson's ratio $v=0.3$. The thickness was increased to $112.5 \mu \mathrm{m}$ to model the corner reinforcements. The steel rods attached to the edge of the tabs were modeled as beams with a circular cross section, with a radius of $1.5 \mathrm{~mm}$ and Young's modulus of $210,000 \mathrm{MPa}$
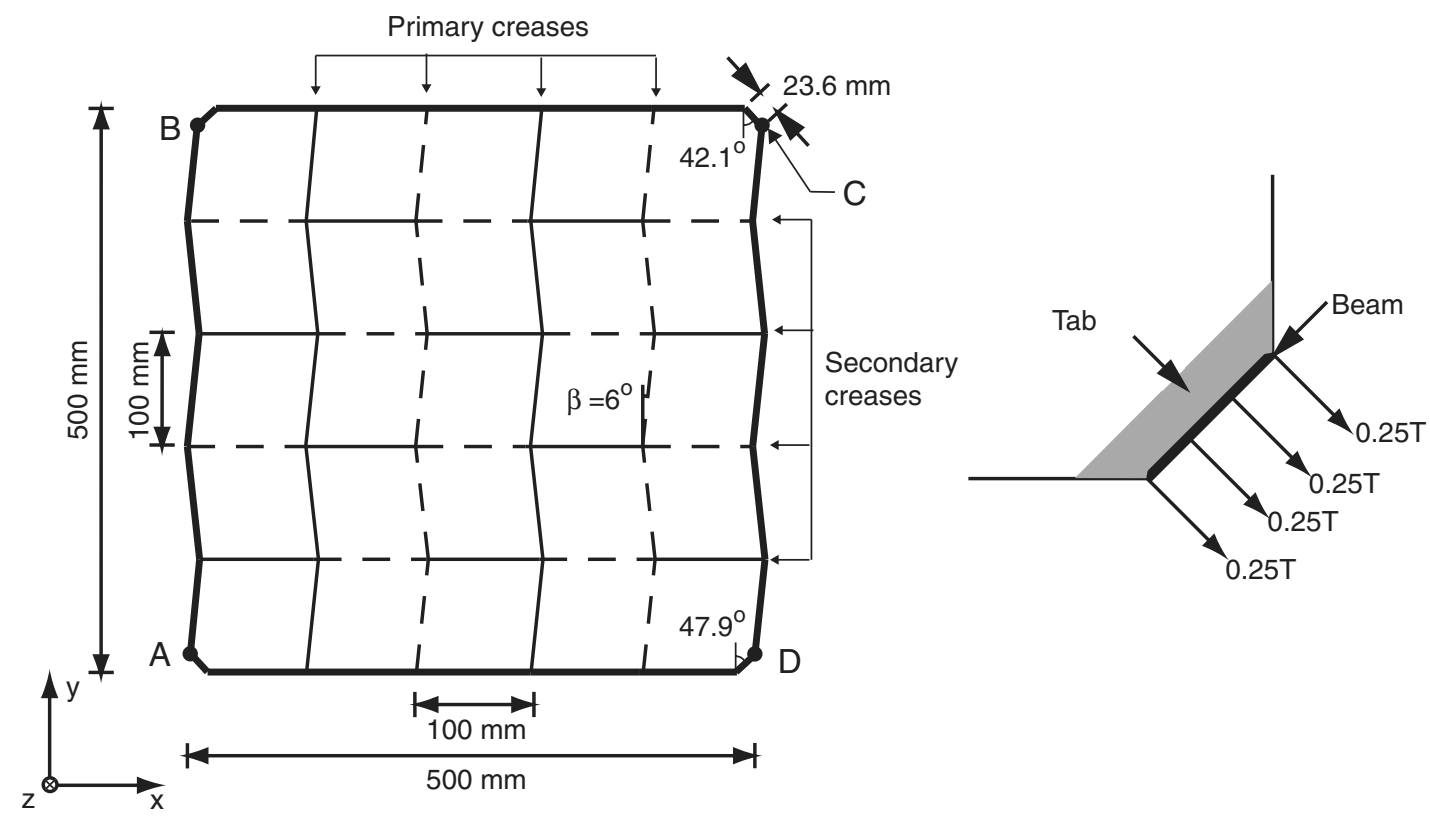

a) Geometry

Fig. 5 Finite element model.

b) Trimmed corner detail 


\section{Load Levels}

The maximum value of the corner force $T$, see Fig. $5 \mathrm{~b}$, was $0.2 \mathrm{~N}$. This value corresponds to an average stress level of about $20 \mathrm{kPa}$ in an uncreased membrane of equal dimensions to that investigated. This stress magnitude is of the same order of prestress levels that are currently planned for solar-sail missions.

\section{Geometric Imperfections}

Geometric imperfections were seeded into the model to facilitate the formation of buckles (wrinkles). Random imperfections $z$ were seeded [8]

$$
z=\alpha \zeta_{i} h \quad(i=1, \ldots, N)
$$

where $\alpha$ is a dimensionless amplitude parameter, $\zeta_{i} \in[-1,1]$ is a pseudorandom number, $h$ is the membrane thickness, and $N$ is the total number of nodes in the model. A MATLAB script was written to generate a table of nodal geometric imperfections that were subsequently superposed to the heights of all of the nodes of the finite element model. The value $\alpha=0.2$ was used [8].

\section{E. Analysis Procedure}

A geometrically nonlinear analysis was carried out using the ABAQUS *NLGEOM command, with automatic stabilization through the use of *STATIC, STABILIZE. The default stabilization factor in ABAQUS is too large to achieve accurate results. It is desirable to use the smallest stabilization factor possible, to reduce the amount of numerical damping in the model, but the computational expense of running simulations quickly increases with stabilization factors below a certain threshold. Values between $6 \times 10^{-7}$ and $1 \times 10^{-6}$, intermediate between those used in [ㅇ-ㅁ] , were used.

\section{F. Sensitivity Study}

Three different finite element models were used to test the sensitivity of the finite element solution with respect to different combinations of mesh resolution and stabilization factor. A low resolution mesh of $90 \times 90$ elements was tested with stabilization factors of $6 \times 10^{-7}$ and $1 \times 10^{-6}$. A high-resolution mesh of $180 \times$ 180 elements was tested with a stabilization factor of $1 \times 10^{-6}$, and this simulation took nine days on a $3.4 \mathrm{GHz}$ Pentium 4 PC. There was insufficient time available to test the high-resolution mesh with the lower stabilization factor.

Figure 7 shows plots of the surface profile obtained from these three models. These profiles are obtained by intersecting the midplane of five consecutive panels of the membrane, at a distance of about $5 \mathrm{~mm}$ from a central primary crease.

The figure shows that there is a tradeoff between mesh resolution and stabilization factor, and the comparison indicates that for the purpose of modeling the membrane's surface, a higher mesh

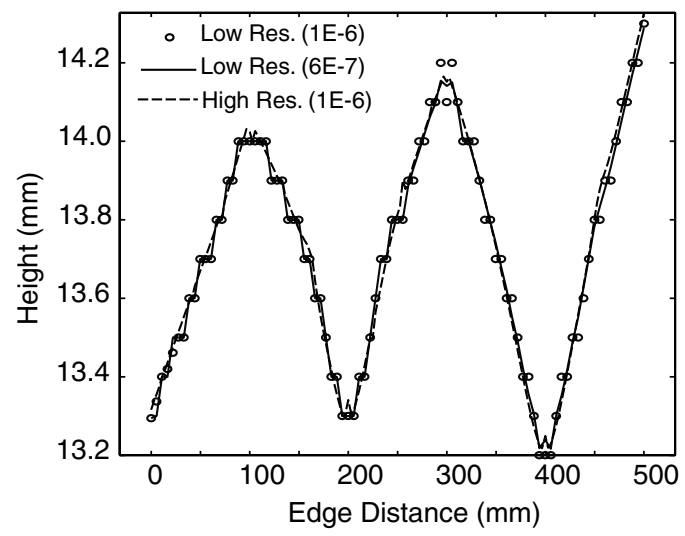

Fig. 7 Comparison of surface profiles for different combinations of mesh resolution and stabilization factor along section parallel to a primary crease.

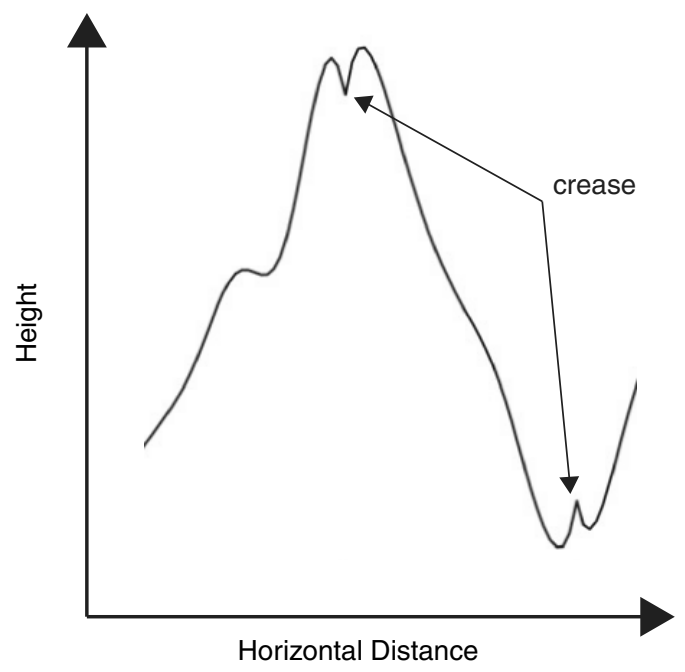

Fig. 8 Detail of surface profile across two secondary creases, showing curvature reversal effect.

resolution is preferable to the use of a lower stabilization factor. The low-resolution mesh is unable to accurately capture the curvature reversal of the membrane near the creases, as this effect occurs on a very short wavelength. Figure $\underline{8}$ shows a detailed view of a typical surface profile, obtained from a high-resolution model. Similar comparisons of the stress variation near the creases confirm that it is preferable to use a higher resolution mesh rather than a lower stabilization factor, as this will allow the model to capture higher levels of stress in the vicinity of the creases. However, the general shape of the plots is very similar and this indicates that low-resolution models would also be acceptable if detailed knowledge of the stress peaks is not essential. In light of this sensitivity analysis, a highresolution model with a stabilization factor of $1 \times 10^{-6}$ was selected as the basis for all finite element simulations.

\section{G. Results}

During the unfolding of an ideal Miura-ori surface, the displacements in the $x$ direction dominate over the displacements in the $y$ direction toward the end of deployment. This happens also here, with the maximum nodal displacement in the $x$ direction predicted to be 87.7 times that of the maximum nodal displacement in the $y$ direction, as shown in Fig. 9. Furthermore, the component of the displacement field in the $x$ direction displays a uniform gradient, which is also what would be expected for an ideal Miura-ori surface.

The load-displacement history of point $\mathrm{C}$, near the right corner (see Fig. 5), is shown in Fig. 10. The membrane stiffens considerably after a displacement of about $13 \mathrm{~mm}$, when the corner loads are approximately $0.02 \mathrm{~N}$. This behavior is similar to a trend observed by Murphey [4] for randomly wrinkled membranes.

Localized buckling of the membrane occurs between the creases and is clearly visible in Fig. 11. Looking carefully at the loaddisplacement relationship for a corner, see the insert in Fig. 10, one finds a large number of nearly horizontal jumps. These discontinuities are believed to be associated with mode jumps, already known to occur in uncreased membranes [7], and hence are associated with (small) changes in the wrinkle pattern in the membrane. It has been found that changing the value of the stabilization factor used in the simulation has the effect of changing the precise value of the load at which these jumps occur, but does not change fundamentally the type of response. Plots of the surface profile indicate that the amplitudes of the buckles between the creases is small in relation to the amplitudes of the creases. An interesting feature that has been observed is the snap-through of the nodes located on the secondary creases, which does not happen for the nodes located on the primary creases.

To understand how a creased membrane carries loads, the midplane normal stress components have been plotted over a narrow 


\section{$+13.6$ \\ $+10.1$ \\ $+6.7$ \\ $+3.2$ \\ $-0.2$}

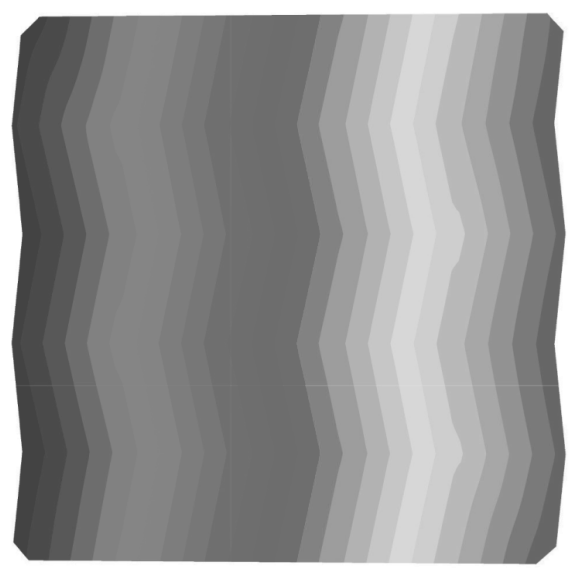

a)

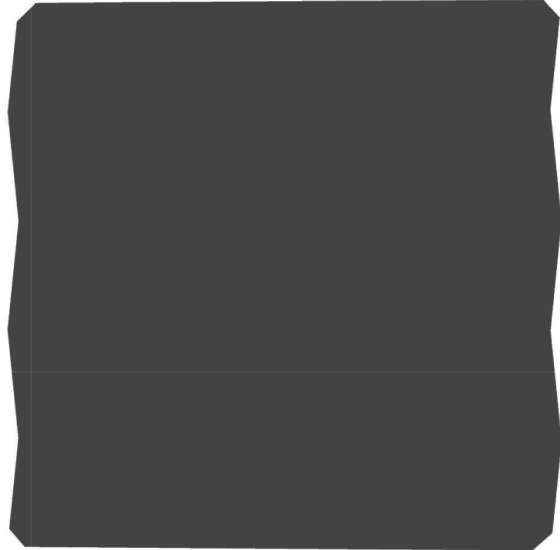

b)

Fig. 9 Displacement components, in millimeters, in a) $x$ direction and b) $y$ direction.

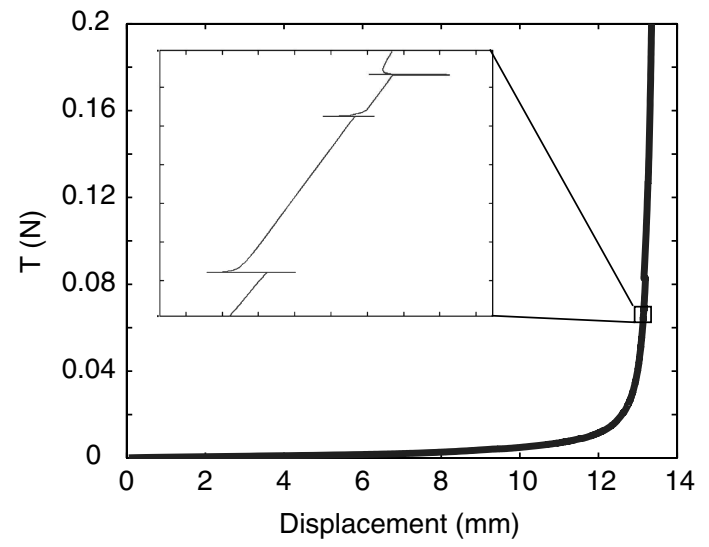

Fig. 10 Load-displacement relationship for corner $\mathrm{C}$ (insert shows magnified response over a very small displacement range).

range, in Fig. 12. The figure shows that the midplane stresses are generally quite small, i.e., of the same order as in an uncreased membrane, but tend to be two or three times larger near the creases. Even larger values occur near the four corners of the membrane.

However, this is by no means the full picture, as much larger bending stresses occur along the creases. Figure 13 shows the distribution of the midplane curvatures in the membrane. The largest value is $0.059 \mathrm{~mm}^{-1}$, in the $x$ direction, which corresponds to a maximum bending strain of $0.07 \%$, and hence to a maximum normal stress component of $2.7 \mathrm{MPa}$. Note that this value is about one-tenth of the yield stress of Kapton.

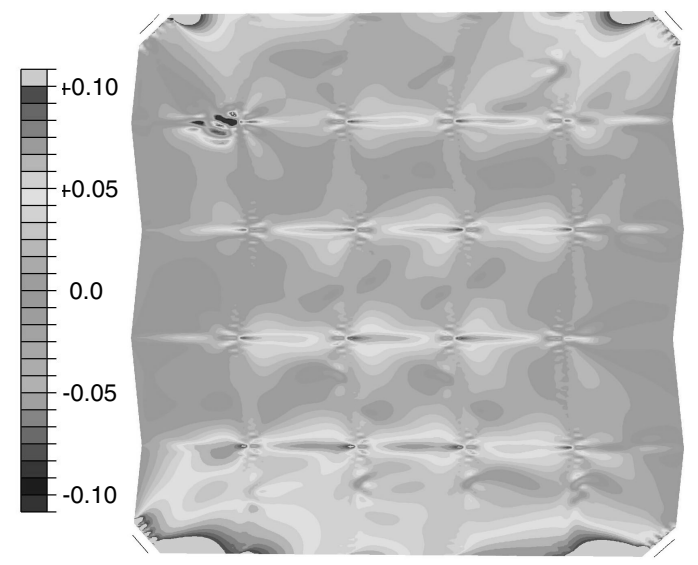

a)

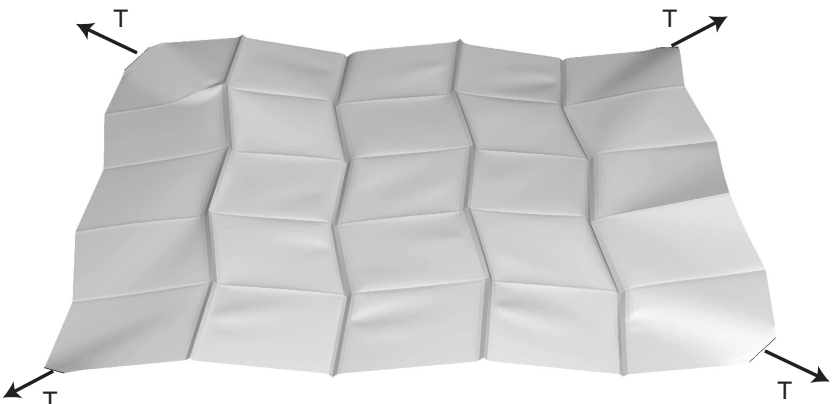

Fig. 11 Deformed membrane, with out-of-plane deformations amplified by a factor of 10 .

In conclusion, a membrane with systematic creases arranged according to the Miura-ori pattern carries loads mainly by means of in-plane action, with associated midplane stresses throughout the membrane and midplane stress concentrations along the creases, plus bending action, concentrated along the creases, that induces much larger stresses, typically an order of magnitude higher.

\section{Comparison of Experimental and Finite Element Results}

In the finite element simulation, even a small set of corner forces induces a corresponding displacement of the corners, but in the experiment, due to friction between the membrane and the supporting plate, a threshold has to be reached before any

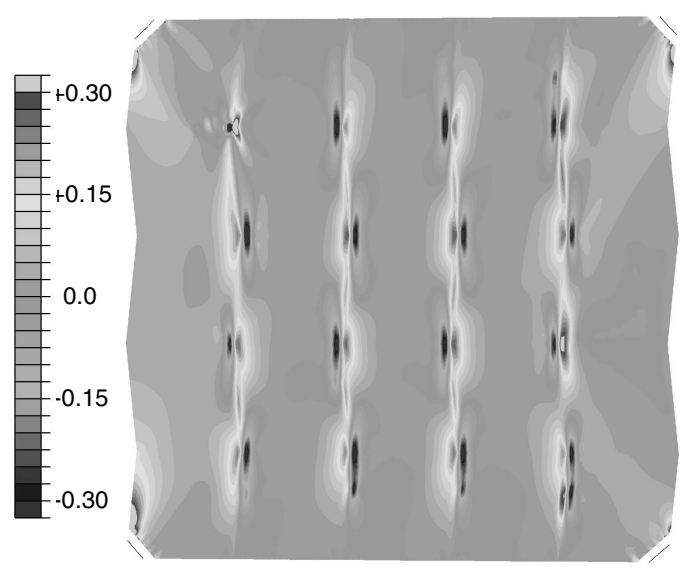

b)

Fig. 12 Midplane normal stress components, in MPa, in a) $x$ direction and b) $y$ direction. 


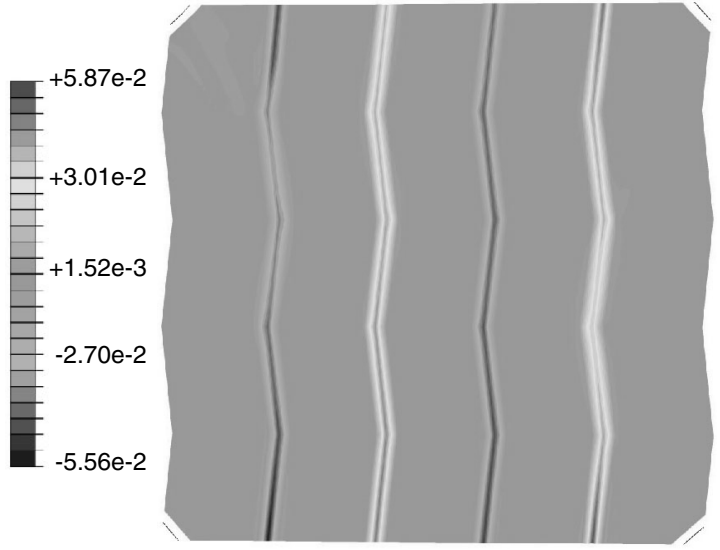

a) $\mathbf{x}$ direction

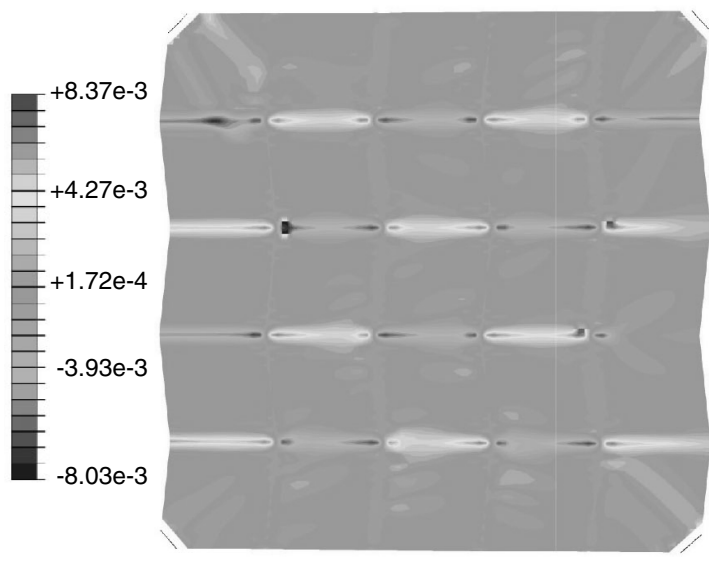

b) y direction

Fig. 13 Midplane curvatures, in $\mathbf{m m}^{-1}$.

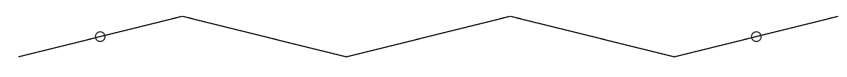

a) Unloaded

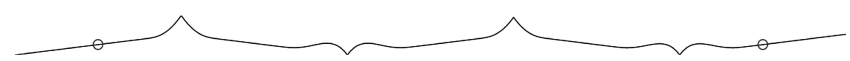

\section{b) Gravity loaded}

Fig. 14 Creased-beam model. The vertical displacements in Fig. 14b are amplified by a factor of 10 .

displacement is observed. The self-weight of the membrane is $0.11 \mathrm{~N}$; the static friction coefficient between Kapton and steel was measured to be 0.19 . Multiplying the weight by the friction coefficient, and dividing by four, to account for the fact that four corner forces are applied simultaneously to the membrane, we obtain a rough estimate, $0.0052 \mathrm{~N}$, of the friction-related threshold for the corner forces. This estimated value is rather small in comparison with the range of forces of interest, and hence it can be concluded that friction effects are negligibly small.

A more significant effect of gravity loading on the membrane is to change the initial shape of the membrane and thus cause an initial set of corner deflections. To fully take into account this effect, one should apply a distributed gravity loading in the finite element model, but this would have the effect of considerably increasing the simulation time. An alternative, quicker approach was adopted instead. This consisted in setting up a two-dimensional finite element creased-beam model of the membrane, of the same kind as the analytical model described in Sec. VI, but using B23H beam elements. At the two kinks that face downward, which model the creases that are in contact with the steel plate, the vertical displacement was set to zero and frictional force components were applied horizontally. A geometrically nonlinear analysis was then carried out in ABAQUS, to determine the horizontal displacements of the end of the creased beam (see Fig. 14).

The deflection thus obtained was then used to estimate an equivalent corner force $T_{0}$ that would cause the same corner displacement in the finite element model of a membrane that is not subject to gravity. The value of this force is $0.034 \mathrm{~N}$. Hence an initial offset of $T_{0}=0.034 \mathrm{~N}$ was applied to the load-displacement curve predicted by the finite element analysis, see Fig. 15 , to compare these results with the experimental measurements, also shown in the figure. The two sets of results are in very good agreement and within the estimated experimental error.

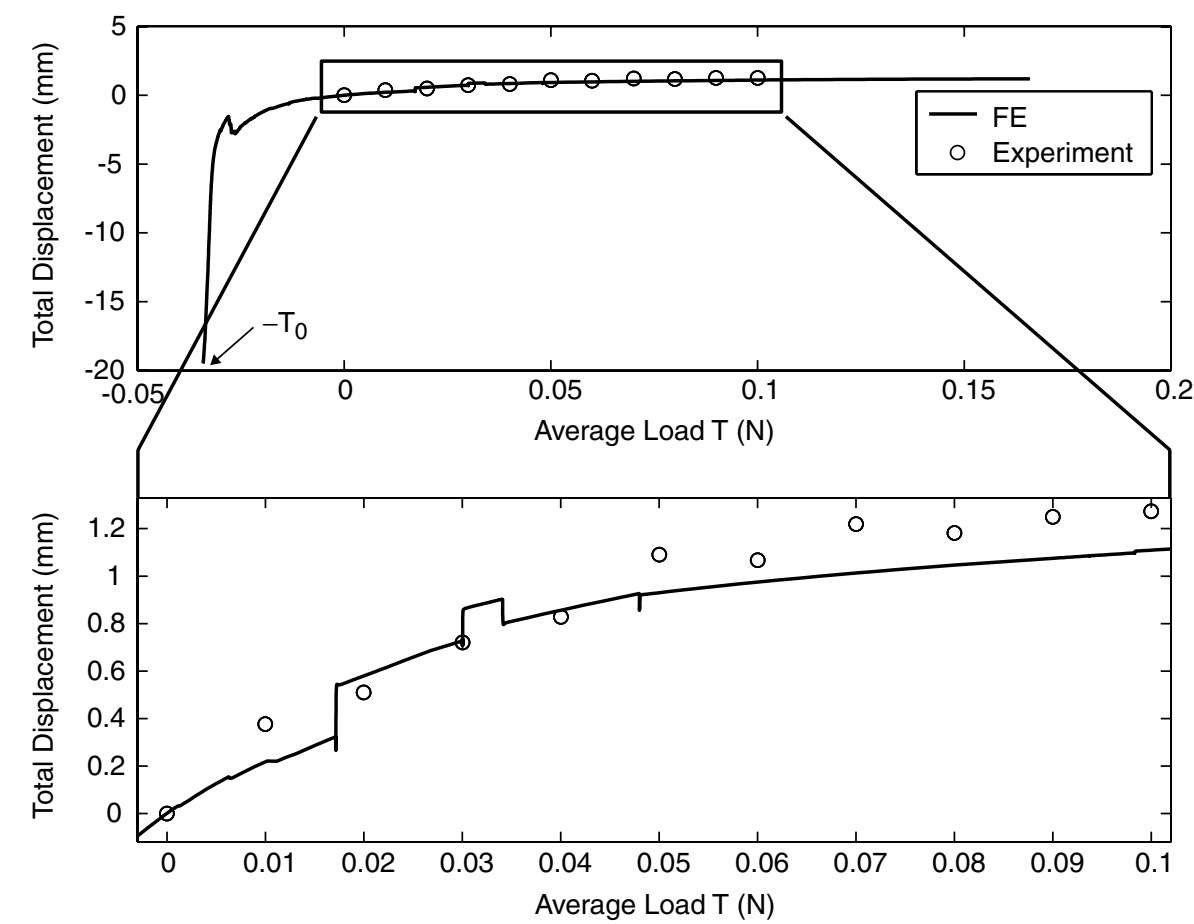

Fig. 15 Comparison of total diagonal displacements from finite element simulation and experimental measurements. 
The discontinuities present in the finite element load-displacement predictions are likely to be the result of a combination of out-of-plane displacements due to localized buckling and mode jumping instabilities.

\section{Analytical Model}

Murphey [4] has proposed a number of analytical models that capture the in-plane load-displacement response of randomly creased membranes. Here we adopt a simplified version of one of Murphey's models and show that it predicts with good accuracy the overall behavior of a membrane with creases arranged according to the Miura-ori pattern.

We make the following three assumptions. First, based on the observation that the maximum stresses in the membrane are well below the yield stress of the material, linear-elastic material behavior is assumed. Second, stretching of the membrane midplane is neglected, and so the only deformation mode that is considered is bending. Third, based on the displacement fields that have been observed, see Fig. 9, it is assumed that parallel edges of the membrane remain parallel throughout, and hence that the behavior of the creased membrane can be captured using only two global kinematic variables $d_{x}$ and $d_{y}$ defined in Fig. 16. The model consists of two uniform creased beams, parallel to the $x$ and $y$ axes.

\section{A. Creased-Beam Model}

Consider the creased-beam ABC shown in Fig. 17. Our aim is to determine the deflection-force relationship for this beam, $\delta=\delta(P)$, as a function of the length $L$, rise $a_{0}$, and flexural stiffness $E I$ of the beam. The beam is assumed to be inextensional. Once $\delta$ is known, it can be used to determine the average longitudinal strain along AC, which can then be used to characterize the behavior of the creased membrane in the $x$ or $y$ direction.

To avoid the slope discontinuity at $\mathrm{B}$, we will analyze only $\mathrm{AB}$, subject to appropriate boundary conditions at B. Denoting by $w(\xi)$ the deflection of the beam, which includes the initial deflection $w_{0}=2 a_{0} \xi / L$ and the additional, elastic deflection $w_{1}$ due to $P$, the bending moment is given by

$$
M=P w=P\left(\frac{2 a_{0} \xi}{L}+w_{1}\right)
$$
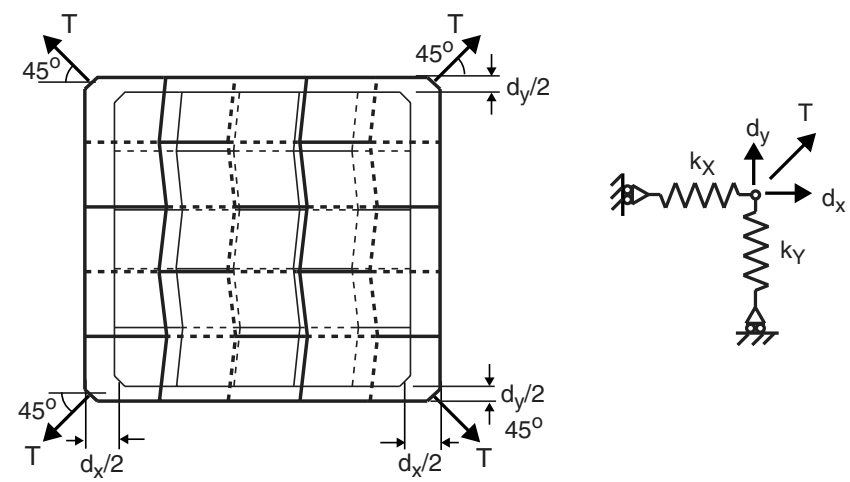

a) Deformation

b) Simple model

Fig. 16 Membrane deformation due to loads $T$ (thicker lines) and its initial configuration for $T=0$ (thinner lines), and the simple twoparameter model.
Substituting the standard moment-curvature relationship for small rotations

$$
M=E I \frac{d^{2} w_{1}}{d \xi^{2}}
$$

we obtain

$$
\frac{d^{2} w_{1}}{d \xi^{2}}=\frac{P}{E I}\left(\frac{2 a_{0} \xi}{L}+w_{1}\right)
$$

This equation can be simplified by introducing the nondimensional ratio between $P$ and the Euler buckling load of a straight beam of length $L$

$$
\alpha=\frac{L}{\pi} \sqrt{\frac{P}{E I}}
$$

Substituting Eq. (ㅁ) into Eq. (7) and rearranging gives

$$
\left(\frac{L}{\pi \alpha}\right)^{2} \frac{d^{2} w_{1}}{d \xi^{2}}-w_{1}=\frac{2 a_{0}}{L} \xi
$$

The solution of this equation, having included the boundary conditions $w_{1}=0$ at $\xi=0$ and $d w_{1} / d \xi=0$ at $\xi=L / 2$, is

$$
w_{1}=\left(\frac{2 a_{0}}{\pi \alpha}\right) \frac{\sinh (\pi \alpha \xi / L)}{\cosh (\pi \alpha / 2)}-\frac{2 a_{0}}{L} \xi
$$

Hence the deflected shape of the beam is given by

$$
w=w_{0}+w_{1}=\left(\frac{2 a_{0}}{\pi \alpha}\right) \frac{\sinh (\pi \alpha \xi / L)}{\cosh (\pi \alpha / 2)}
$$

Next we want to determine the end displacement of the beam $\delta$. This can be found by integrating the strain along $\xi$ due to $w$ and then subtracting the initial displacement due to creasing, $-2 a_{0}^{2} / L$. This gives

$$
\delta=2 \int_{0}^{L / 2}\left(\frac{\mathrm{d} u}{\mathrm{~d} \xi}\right) \mathrm{d} \xi+\frac{2 a_{0}^{2}}{L}
$$

where $\mathrm{d} u / \mathrm{d} \xi$ can be found by noting that the Green strain $\epsilon_{G}$ along $\xi$ has to vanish in an inextensional beam, hence

$$
\epsilon_{G}=\frac{d u}{d \xi}+\frac{1}{2}\left(\frac{d w}{d \xi}\right)^{2}=0
$$

Substituting

$$
\frac{d u}{d \xi}=-\frac{1}{2}\left(\frac{d w}{d \xi}\right)^{2}
$$

into Eq. (12), differentiating $w$ in Eq. (11), and then solving the integral, we obtain

$$
\delta=\left(2-\frac{\sinh (\pi \alpha)+\pi \alpha}{\pi \alpha \cosh ^{2}(\pi \alpha / 2)}\right) \frac{a_{0}^{2}}{L}
$$

Then, dividing by $L$, we find the average strain along an equivalent, straight beam of length $L$

$$
\epsilon=\frac{\delta}{L}=\left(2-\frac{\sinh (\pi \alpha)+\pi \alpha}{\pi \alpha \cosh ^{2}(\pi \alpha / 2)}\right) \frac{a_{0}^{2}}{L^{2}}
$$

\section{B. Validation of Analytical Model}

Predictions of the load-displacement behavior of node $\mathrm{C}$, defined in Fig. 5, from our simple analytical model are compared with the results of a finite element simulation for a massless, initially creased membrane.

First, we computed the average strains in the two creased beams $\bar{\epsilon}_{x}$ and $\bar{\epsilon}_{y}$, using Eqs. (ㅇ) and (16). It was assumed that $P=T / \sqrt{2}$, i.e., 


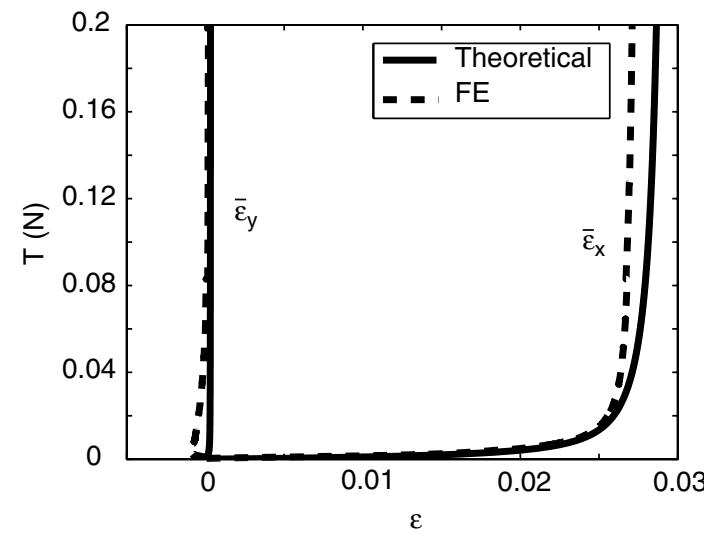

a) Average strains

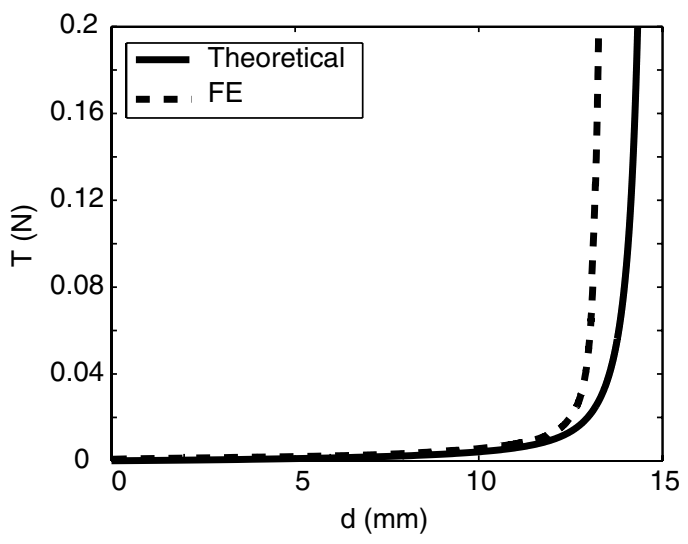

b) Corner displacements

Fig. 18 Prediction from theoretical model and finite element analysis.

each corner force is shared equally between the two creased beams, and $T$ varies between 0 and $0.2 \mathrm{~N}$. The bending stiffness of the creased beams was computed assuming a rectangular cross section beam with the breadth and thickness equal to the full width and thickness of the membrane, respectively. Hence

$$
E I=3530 \times \frac{500\left(25 \times 10^{-3}\right)^{3}}{12}=2.298 \mathrm{~N} \mathrm{~mm}^{2}
$$

We will denote quantities that refer to the creased beams parallel to the $x$ axis and the $y$ axis with the subscripts $x$ and $y$, respectively. Note that in Fig. 2b, the projected length $L$ of the creased beam corresponds to $\overline{O A} / 2$ for the calculation of $\bar{\epsilon}_{x}$ and $\overline{O B} / 2$ for the calculation of $\bar{\epsilon}_{y}$. Hence, substituting $\theta=\theta_{0}=13.8 \mathrm{deg}$ and $\overline{O A}_{\max }=\overline{O B}_{\max }=2 l=200 \mathrm{~mm}$, we obtain $L_{x}=97.14 \mathrm{~mm}$ and $L_{y}=99.98 \mathrm{~mm}$, respectively. Note that $l(=100 \mathrm{~mm})$ denotes the width and height of each panel of the Miura-ori pattern.

Finally, the crease heights in the two directions $a_{0 x}$ and $a_{0 y}$ can be obtained from Pythagoras' theorem, given the projected lengths $L_{x}$ and $L_{y}$

$$
a_{0 x}=\sqrt{l^{2}-L_{x}^{2}} / 2=11.9 \mathrm{~mm} \quad a_{0 y}=\sqrt{l^{2}-L_{y}^{2}} / 2=1.3 \mathrm{~mm}
$$

Figure 18a shows the variation of $\bar{\epsilon}_{x}$ and $\bar{\epsilon}_{y}$ with $T$; the corresponding average strains derived from a finite element solution are shown for comparison. Figure $18 \mathrm{~b}$ shows the variation of the total corner displacement $d$ with $T$.

Compared with the finite element model, the theoretical model overestimates the final strain in the $x$ direction and the total corner displacement by less than $6 \%$. The difference in final strains in the $y$ direction is proportionally much larger, but their magnitude is actually so small to have practically no effect on the accuracy of the predicted corner displacement.

Also note that the average strain in the $y$ direction, predicted by the finite element model, shows an initial decrease, which is not captured by the theoretical model. This is not surprising, as no coupling between the two creased beams has been included in the model.

\section{Discussion and Conclusions}

It has been shown that a membrane creased according to the Miura-ori folding pattern will partially deploy until it reaches an equilibrium configuration. Taking this configuration as a starting point, the response of the membrane to two pairs of equal and opposite diagonal corner forces has been investigated. The magnitude of the forces was such that they would generate stresses on the order of $0.01 \mathrm{MPa}$ in an unwrinkled membrane.

A finite element simulation of a systematically wrinkled, $25 \mu \mathrm{m}$ thick Kapton membrane, modeled with shell elements in the ABAQUS finite element package, has been successfully carried out.
The initial geometry of the structure was defined on the basis of an experimentally measured deployment angle; random geometric imperfections were seeded on this initial geometry. An automatically stabilized, geometrically nonlinear analysis was performed under load control. It was shown that a fine mesh is required to correctly predict the membrane's deformed surface shape, including the localized buckles that are observed in practice, and the peak magnitudes of the tensile and compressive stresses in the membrane.

The load-displacement response is highly nonlinear, with considerable stiffening taking place when the shortening of the membrane associated with creasing has been almost fully taken up. Plots of the stress distribution, obtained from the ABAQUS analysis, indicate that the primary load-carrying mechanism is in-plane stretching of the flat parts of the membrane, coupled with out-ofplane bending of narrow crease regions; hence the corner deformation is essentially controlled by the much softer bending mode.

Experimental measurements of the average primary crease angle have shown that it remains almost constant over the load range of interest. Hence, it is reasonable to model the material behavior as linear elastic and, upon offsetting the finite element loaddisplacement curve for the effects of gravity, an excellent level of agreement was obtained between the simulated finite element response and the experimental measurements.

A simple analytical model of the creased membrane has been developed, consisting of two perpendicular creased beams whose geometric properties are related to physical dimensions of the membrane. It was found that this model is able to predict the relative displacements of the corners with very good accuracy.

There are no obvious limitations regarding the applicability of the analytical results to much larger membranes.

\section{Acknowledgment}

We thank C. R. Steele for discussing aspects of the analytical model.

\section{References}

[1] Miura, K., "Method of Packaging and Deployment of Large Membranes in Space," 31st IAF Congress, International Astronautical Federation, Paris, 1981, pp. 1-10.

[2] Miura, K., and Natori, M., "2-D Array Experiment on Board a Space Flyer Unit," Space Solar Power Review, Vol. 5, No. 4, 1985, pp. 345356.

[3] Horner, G. C., and Elliot, M. D., "A Fabrication and Deployment Approach for a Miura-Ori Solar Sail Model," 43rd AIAA/ASME/ASCE/ AHS/AHS Structures, Structural Dynamics and Materials Conference, AIAA Paper 2002-1708, 2002.

[4] Murphey, T. W., "A Nonlinear Elastic Constitutive Model for Wrinkled Thin Films," Ph.D. Dissertation, Univ. of Colorado, Boulder, CO, 2000. 
[5] Wong, Y. W., and Pellegrino, S., "Wrinkled Membranes, Part 1: Experiments," Journal of Mechanics of Materials and Structures, Vol. 1, No. 1, 2006, pp. 1-23.

[6] Wong, Y. W., Pellegrino, S., and Park, K. C., "Prediction of Wrinkle Amplitudes in Square Solar Sails," 44th AIAA/ASME/ASCE/AHS/ASC Structures, Structural Dynamics and Materials Conference, AIAA Paper 2003-1982, 2003.

[7] Wong, Y. W., and Pellegrino, S., "Wrinkled Membranes, Part 3: Numerical Simulations," Journal of Mechanics of Materials and Structures, Vol. 1, No. 1, 2006, pp. 61-93.

[8] Tessler, A., Sleight, D. W., and Wang, J. T., "Effective Modeling and Nonlinear Shell Analysis of Thin Membranes Exhibiting Structural
Wrinkling," Journal of Spacecraft and Rockets, Vol. 42, No. 2, 2005, pp. 287-298.

[9] Gough, A., Hossain, N. M. A., Jenkins, C. H., Blandino, J. R., and Hendricks, A., "Experimental and Numerical Study of Creased Membranes," 46th AIAA/ASME/ASCE/AHS/ASC Structures, Structural Dynamics and Materials Conference, AIAA Paper 2005-1976, 2005.

[10] Wong, Y. W., "Wrinkling of Thin Membrane Structures," Ph.D. Dissertation, Univ. of Cambridge, Cambridge, England, U.K., 2003.

G. Agnes Associate Editor 